\title{
Livin modulates the apoptotic effects of vesicular stomatotitis virus in lung adenocarcinoma
}

\author{
GUI-HONG LIU ${ }^{*}$, WEI-LIN WANG ${ }^{4 *}$, CHUN WANG ${ }^{3}$, CHANG-LIN LI ${ }^{4}$, \\ XIAO-LONG ZHANG ${ }^{2}$, QIU LI ${ }^{2}$ and ZHEN-YU DING ${ }^{1}$ \\ Divisions of ${ }^{1}$ Thoracic Oncology, ${ }^{2}$ Abdominal Oncology, Cancer Center, State Key Laboratory of Biotherapy and \\ ${ }^{3}$ Endocrinology and Metabolism, West China Hospital, West China Medical School, Sichuan University; \\ ${ }^{4}$ Department of Oncology, Chengdu First People's Hospital, Chengdu 610041, P.R. China
}

Received June 30, 2015; Accepted August 10, 2015

DOI: $10.3892 /$ ijo.2015.3183

\begin{abstract}
Vesicular stomatitis virus (VSV) has shown promise in cancer treatment. However, it achieved limited effects against lung cancer. Lung cancer has intrinsic mechanisms that render resistance to VSV. In this study, we attempted to explore the expression of the anti-apoptotic factor Livin in lung adenocarcinoma and its possible relationship to VSV vulnerability. We found VSV induced apoptosis in a time- and dose-dependent manner, with the concomitant change in the expression of Livin. We elevated the expression of Livin both transiently and stably, and the cells became insensitive to VSV treatment. We further found the BIR domain of Livin was mainly responsible for its modulation effects. This finding suggested a possible interaction with the second mitochondria-derived activator of caspase (SMAC). The knock-down of SMAC also inhibited apoptosis by VSV. The relationship was confirmed by the co-immunoprecipitation. Finally, we knocked down the endogenous Livin, and the knock-down sensitized cells to VSV treatment. Our results suggested the important role of Livin and its partner molecule in the process of VSV treatment.
\end{abstract}

\section{Introduction}

Lung cancer is the leading cause of malignancy-related death worldwide. Non-small cell lung cancer constitutes $\sim 80 \%$ of all cases (1). The treatment including surgery, radiotherapy and chemotherapy has changed little for decades. Recently, targeted therapy was confirmed in a subset of patients with

Correspondence to: Professor Zhen-Yu Ding, Division of Thoracic Oncology, Cancer Center, State Key Laboratory of Biotherapy, West China Hospital, West China Medical School, Sichuan University, GuoXue Lane 37, Chengdu 610041, P.R. China

E-mail: dingzhenyu@scu.edu.cn

*Contributed equally

Key words: apoptosis, vesicular stomatitis virus, Livin, second mitochondria-derived activator of caspase, lung cancer epithelial growth factor receptor gene mutation or amplification. However, $<20 \%$ of patients survive for 5 years (2). The search for novel treatment modalities is highly required.

Vesicular stomatitis virus (VSV) from the family of rhabdoviridae is an enveloped, negative-sense RNA virus (3). VSV was suggested to kill selectively a variety of tumor cell lines and proposed as a promising cancer treatment (3-5). We and others tested its efficacy against a panel of both murine and human cancers including ovarian cancer, colorectal cancer, and lung cancer (6-9). Notably, although VSV showed promise in ovarian and colorectal cancer, it achieved limited effects against lung cancer. The lung cancer intrinsic mechanisms render resistance to VSV.

Cancer cells have defects in the interferon (IFN) signaling pathway (10). The defects make the cancer cells vulnerable to viruses including VSV. VSV induces apoptosis in cancer cells that have defects in the IFN pathway (11). The apoptosis pathway plays an important role in mediating the tumoricidal effects of VSV. Livin was discovered as a member of the inhibitor of apoptosis family (12-15). It is expressed in a variety of tumors (16-19), but hardly detectable in the normal tissue (20). Two splicing variants have been identified (designated $\alpha$ and $\beta$ isoforms) for Livin, which are almost identical except for a 54 bp truncation in exon 6 (15). The overexpression of both isoforms block apoptosis induced by TNF- $\alpha$ and anti-CD95 antibody. In this study, we attempted to explore the expression of Livin in lung adenocarcinoma and its possible relationship to VSV vulnerability.

\section{Materials and methods}

Expression plasmids. We previously constructed the expression plasmid for the full length Livin- $\beta$ and the truncated form lacking the first 52 amino acids (tLiv) $(21,22)$. The baculovirus inhibitor of apoptosis protein repeat (BIR) domain of Livin (BIRLiv, amino acid 53-220) was subcloned into the expression plasmid pVITRO with a FLAG tag. The carboxyl terminal of Livin (cLiv, amino acid 221-280) compassing the Really Interesting New Gene domain was subcloned into the expression plasmid p-EGFP-N1 with green fluorescent protein fused at its carboxyl terminal. The expression vector for the second mitochondria-derived activator of caspase (SMAC/Diablo) 
was constructed by cloning its sequence into the parental plasmid pTango-zeo. All the constructs were confirmed by sequencing.

Reagents and cells. Cytotoxic drug cisplatin, caspase-3 inhibitor z-DEVD-fmk, the colorimetric caspase-3 substrate z-DEVDpNA, and antibiotic Geneticin were all purchased from Sigma (Sigma-Aldrich, St. Louis, MO, USA). Stock preparation of the reagents was stored at $-20^{\circ} \mathrm{C}$ until use. A549 human lung adenocarcinoma cells, HeLa cervial cancer cells, MCF-7 breast cancer cells, A2780 ovarian cancer cells, and HCT116 colon cancer cells were all from the American Type Culture Collection. Cells were maintained in Dulbecco's modified Eagle's medium (Sigma-Aldrich) supplemented with 10\% FBS (Invitrogen, Carlsbad, CA, USA), and antibiotics. The production and titration of VSV was performed as we described before (6).

Cell transfection. Cells were transfected by using Lipofectamine 2000 transfection reagent (Invitrogen) according to the manufacturer's instructions. Lipofectamine 2000 was used at a ratio of $2.5 \mu \mathrm{g} / \mu \mathrm{l}$ in input plasmid, and Lipofectin-DNA complexes were incubated with cells for $6 \mathrm{~h}$ at $37^{\circ} \mathrm{C}$. For stable transfection, cells transfected with the expression plasmid or control plasmid (empty vector) were selected in Geneticin $(800 \mu \mathrm{g} / \mathrm{ml})$ for 2 weeks (starting at $24 \mathrm{~h}$ after transfection) and maintained in culture medium supplemented with Geneticin (400 $\mu \mathrm{g} / \mathrm{ml})$.

Western blot analysis. Proteins were resolved by electrophoresis on $10 \%$ polyacrylamide pre-cast gels (Bio-Rad, Hercules, CA,USA), electroblotted to Immobilon-P(Millipore, Shanghai, China) and incubated with block solution (5\% non-fat milk, $0.1 \%$ Tween-20, in TBS). Signals were detected with ECL Plus Western Blot Detection system (GE Healthcare, Beijing, China). The preparation of the rabbit polyclonal anti-Livin primary antibody was described previously $(21,22),(1: 1,000)$. The rabbit monoclonal anti-caspase-3 antibody was from Cell Signaling Technology (9665, Beverly, MA, USA 1:1,000). The rabbit monoclonal anti-SMAC antibody was from Epitomics (1201-1, Burlingame, CA, USA 1:500). The mouse monoclonal antibody for $\beta$-actin and HRP-conjugated secondary antibodies were from OriGene (Beijing, China, 1:5,000 and $1: 8,000$ respectively).

Cell viability. Survival of cells after treatment was quantified by CCK-8 assay (Dojindo, Shanghai, China) according to the manufacturer's instructions. Briefly, cells were seeded in 96-well tissue culture plate, in $100 \mu \mathrm{l}$ culture medium. CCK-8 labeling solution was added and the absorbance was measured at a wavelength of $450 \mathrm{~nm}$ with a microplate reader (Bio-Rad, Hercules, CA, USA).

Apoptosis assay. The apoptosis rate was determined on a flow cytometry with a propidium iodide (PI)-Annexin V double staining kit (KeyGen Biotech, Shanghai, China). Briefly, cells were maintained in complete media. After treatment, cells were washed with PBS and stained with PI and Annexin V. The fluorescence signal was detected on a flow cytometer (FACSCalibre, Becton-Dickinson).
Immunoprecipitation. The immunoprecipitation was performed with an immunoprecipitation kit (Beyotime, Shanghai, China). Briefly, cell lysates were incubated with anti-Livin antibody (1:500) at $4^{\circ} \mathrm{C}$ overnight. The combined complex was immobilized with protein $\mathrm{A}+\mathrm{G}$ agarose, and then the agarose was washed twice with PBS. Finally, the precipitated protein was eluted with elution buffer. The eluted Livin protein was then probed with an anti-SMAC antibody.

Terminal deoxynucleotidyl transferase-mediated dUTP nick end labeling (TUNEL) assay. Cells in a 6-well plate were mounted and sujected to fluorescent in situ TUNEL assay by using an in situ apoptotic cell detection kit (Promega, Madison, WI, USA) according to the manufacturer's protocol. Apoptotic cells were counted under a light microscope in randomly selected fields.

Preparation of whole-cell lysates and cytosolic fraction. For preparation of whole-cell lysates, cells were lysed in RIPA buffer $(150 \mathrm{mM} \mathrm{NaCl}, 2 \%$ Triton X-100, 0.1\% SDS, $50 \mathrm{mM}$ Tris $\mathrm{pH}$ 8.0). For western blot analysis, $1 \%$ protease inhibitor cocktail (Sigma) was also added. For preparation of cytosolic fraction, a nuclear-cytosolic separation kit (Beyotime) was used. Briefly, cells were harvested and pelleted. The pellets were resuspended in Buffer A incubated on ice. Buffer B was added to the solution, and supernatants containing the cytosolic fraction were obtained by centrifugation. Protein concentrations were determined by BCA Protein assay (Thermo Fisher, Woburn, MA, USA).

RNAi knock-down. Cells were transfected with siRNA from Invitrogen. The candidate sequences against Livin were: AUAGAAGGAGGCCAGACGCAACUCC (BIRC7HSS149184), UUUGACCGGAGCAGGAACUGACAGC (BIRC7-HSS149185), and UGCACACUGUGGACAAAGU CUCUUC (BIRC7-HSS149186). The candidate sequences against SMAC were: GGCAGAAGCACAGAUAGAATT (DIABLO-homo-1295), CCGACAAUAUACAAGUUUATT (DIABLO-homo-1013), and CGGUGUUUCUCAGAAU UGATT-3 (DIABLO-homo-900). The scramble siRNA sequence (Invitrogen catalog 12935-300) targeting no known coding sequence was used as a control.

DEVDase activity. The assay was performed on a VersaMAX plate reader coupled with SOFTMAX software (Molecular Devices) operating in the end-point or kinetic mode at $37^{\circ} \mathrm{C}$. DEVDase acitivity was determined by using colorimetric pNA substrates (maximal absorbance at $405 \mathrm{~nm}$ ). Assay buffer was $50 \mathrm{mM}$ Tris, pH 7.4; 0.3\% NP-40, and $10 \mathrm{mM}$ dithiothreitol. Data were recorded every $30 \mathrm{~min}$ for various periods of time as appropriate for each assay.

Real-time PCR. Total RNA was extracted from cells using TRIzol agent (Invitrogen), followed by reverse transcription with an iScript cDNA Synthesis kit (Bio-Rad) according to the manufacturer's instructions. The primers used were: TGACA GAGGAGGAAGAGGAGGAG (Livin, upstream), AGTCAGC GGCCAGTCATAGAAG (Livin, downstream), TTCGATGT GTTCTCTGA (SMAC, upstream), TTGATGTTAAGTCCTG TTG (SMAC, downstream), CCACGAAACTACCTTCAAC 


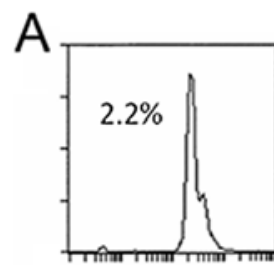

UT

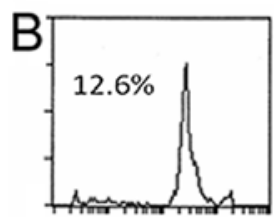

$\mathrm{MOI}=0.001$

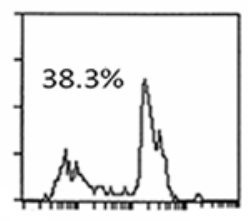

$\mathrm{MOI}=1$

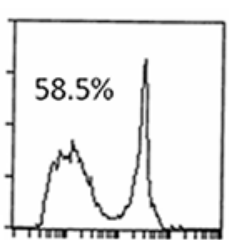

Pt

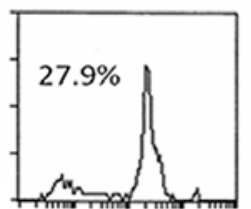

$\mathrm{MOI}=0.01$

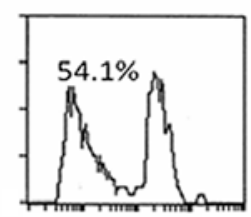

$\mathrm{MOI}=10$

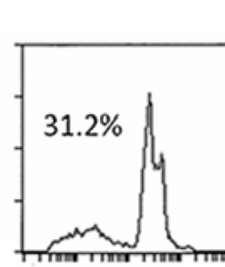

VSV

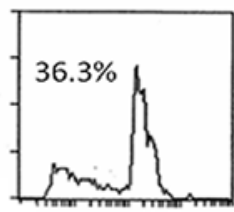

$\mathrm{MOI}=0.1$

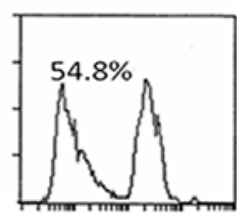

$\mathrm{MOI}=100$

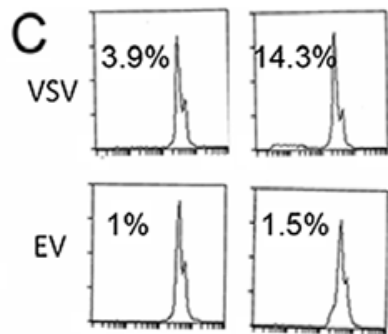

$24 \mathrm{~h}$

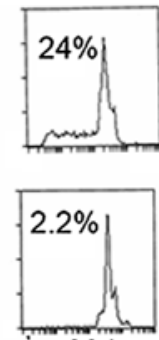

$36 \mathrm{~h}$
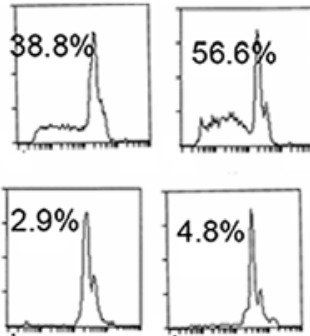

$48 \mathrm{~h}$
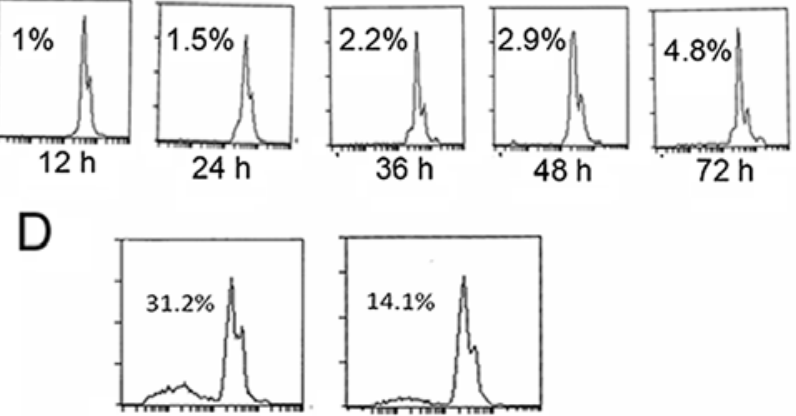

VSV

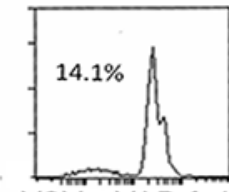

VSV+zVAD-fmk

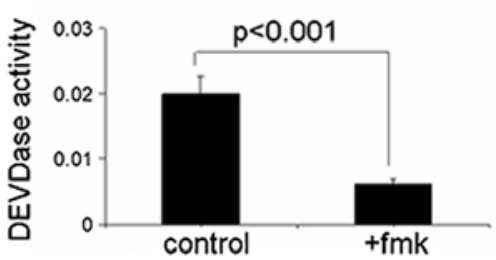

Figure 1. VSV induced death of A549 cells compared with untreated (UT) controls, as evidenced by the presence of sub-G1 peak in FCM (A). The cells treated with cisplatin $(\mathrm{Pt})$ was included as a positive control. With the increasing amount of VSV, the cell death rate increased together (B). As the time elapsed, the cell death also became more significant (C). The pre-treatment of DEVDase inhibitor significantly inhibited the VSV-induced cell death (D).

TCC ( $\beta$-actin, upstream), and GTGATCTCCTTCTGCATCC TGT ( $\beta$-actin, downstream). The PCR mix was set up with a SsoFast EvaGreen Supermix (Bio-Rad). The final concentration of primers was $500 \mathrm{nM}$ each reaction. Real-time PCR reaction was performed on the CFX96 qPCR systems (Bio-Rad). The PCR reaction constituted 40 thermal cycles of $95^{\circ} \mathrm{C}$ for $5 \mathrm{sec}$, and $60^{\circ} \mathrm{C}$ for $5 \mathrm{sec}$. The expression of Livin or SMAC was calculated with the Gene Expression Macro (version 6.0) software (Bio-Rad).

Statistical analysis. Statistical analysis was performed with SPSS 19.0 software (IBM inc.). To evaluate statistical significance the Student's t-test was performed. Results are given as means standard deviations. All two-sided p-values $<0.05$ were considered statistically significant.

\section{Results}

The apoptotic effects of VSV on A549 cells. We tested the apoptotic effects of VSV in vitro and we found VSV induced cell death though to a lesser extent than the cytotoxic reagent cisplatin which was included as a positive control (Fig. 1A). In addition, the effects were both dose- and time-dependent. Transfection at the multiplicity of infection (MOI) of 100 caused death in half of all cells (Fig. 1B). At the MOI of 1, the cell death increased from $3.9 \%$ at $12 \mathrm{~h}$ post-transfection to $56.6 \%$ at $78 \mathrm{~h}$ (Fig. 1C). We explored the relationship between caspase activity and death-inducing effects of VSV. Notably, inhibition of caspase-3 with an exogenous inhibitor (zVAD-fmk) significantly abrogated the death-inducing effects of VSV (Fig. 1D). These results indicated the death-inducing effects of VSV were caspase-dependent.
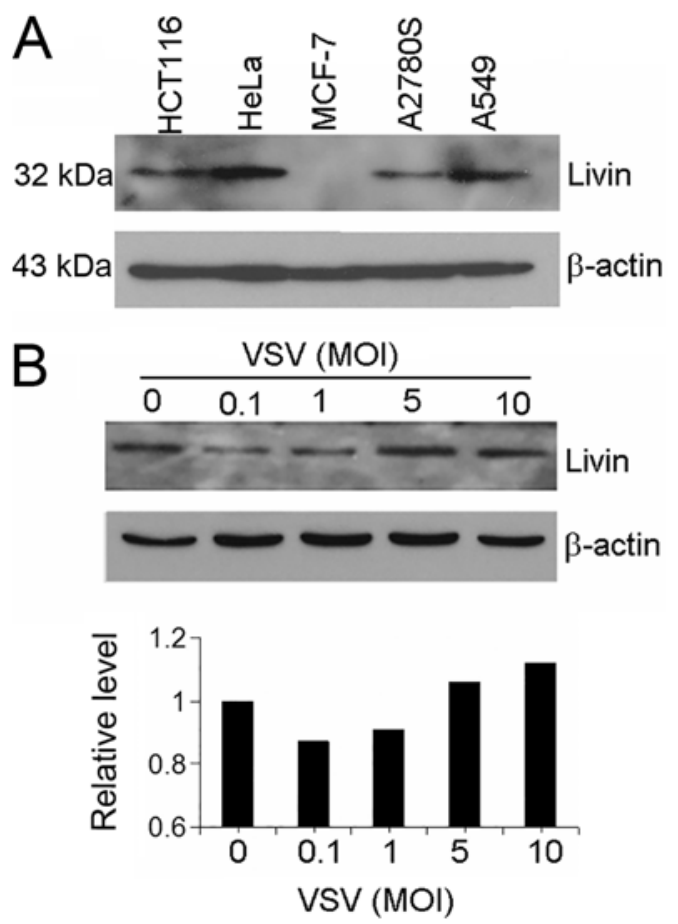

Figure 2. The expression of Livin was detected in a panel of cancer cells including A549 but not in MCF-7 cells (A). The treatment of low dose VSV caused a decreased level of Livin, while higher dose of VSV induced the expression of Livin (B).

The endogenous expression of Livin in A549 cells and its relationship with VSV treatment. The expression of Livin in various cancer cells were detected with western blot analysis (Fig. 2A). 

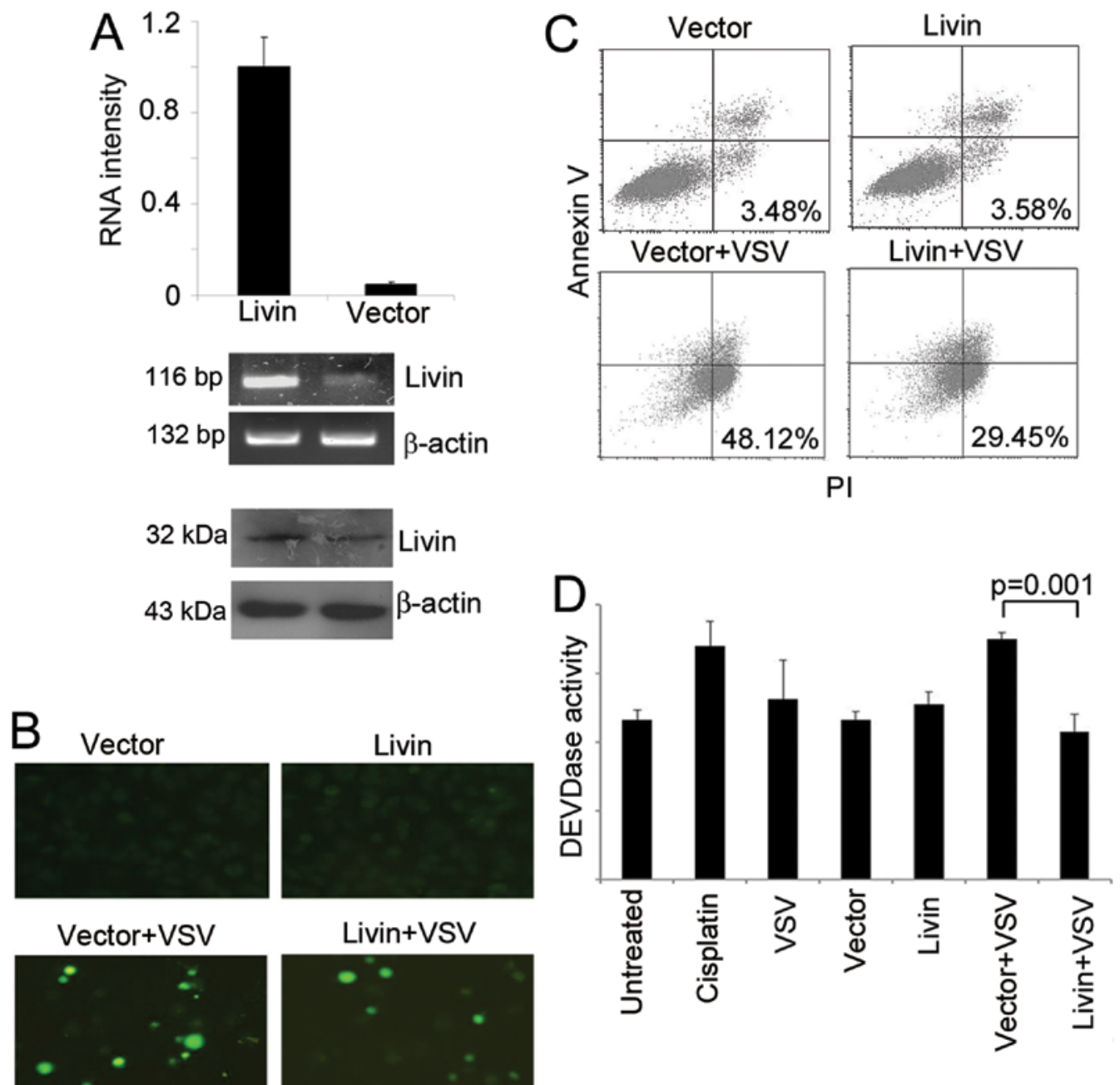

Figure 3. Forced expression of Livin was confirmed by real-time PCR, semi-quantitative PCR, and western blot analyses (A). Overexpression of Livin led to less VSV-induced death, as shown by the TUNEL staining (B). Overexpression of Livin led to reduced cell death from $48.12 \%$ (vector control) to $29.45 \%$ by FCM analysis (C) and less DEVDase activity (D).

Among the cancer cells, the HeLa and A549 cells contained high expression level of Livin, while the MCF-7 breast cancer cells had barely any expression. Then we asked whether the transfection of VSV had effects on Livin expression. At low MOI (MOI=0.1), the transfection downregulated the expression of Livin below the baseline level. The expression became stronger with the increasing dose of VSV (MOI from 0.1 to 10 , Fig. 2B).

Overexpression of Livin and its fragments render A549 cells resistant to VSV-induced cell death. We exogenously elevated the expression of Livin by transfection of an expression plasmid of full-length Livin. The overexpression was confirmed by real-time PCR, half-quantitative PCR, and western blot analysis (Fig. 3A). The overexpression made the cells more resistant to VSV treatment, as showed by qualitative TUNEL staining, and quantitative flow cytometry (Fig. 3B and C). The overexpression also decreased the caspase- 3 activity when challenged with VSV (Fig. 3D). To further confirm the observation, we made stable transfectants of Livin (Fig. 4A). Similar observations were obtained as the transient transfection (Fig. 4B-D).
We next asked which domain was responsible for the anti-apoptotic effects. We prepared several vectors for the different segments of Livin (Fig. 5A). When introduced into cells, the vector for the BIR domain exerted an anti-apoptotic effects together with the full-length Livin (Fig. 5B). The other segments had no obvious effects on the VSV sensitivity. These observations suggested a possible role of the BIR domain for the anti-apoptotic effects of Livin against VSV challenge.

Livin inhibits the apoptotic effects of VSV by binding SMAC in the cytosol. We observed Livin modulated the apoptotic effects of VSV through the inhibition of caspase activity, and the BIR domain was critical for the anti-apoptotic effects. We reasoned that Livin inhibited caspase activity due to SMAC binding with its BIR domain, which is a possible mechanistic explanation for its anti-apoptotic effects. Firstly, we tested the possible involvement of SMAC in the VSV treatment. SMAC was efficiently knocked down with a selected siRNA sequence (Fig. 6A), and the cells became resistant to VSV challenge (Fig. 6B and C). This observation was alike to that of the overexpression of Livin and suggested a possible relationship between SMAC and Livin. 

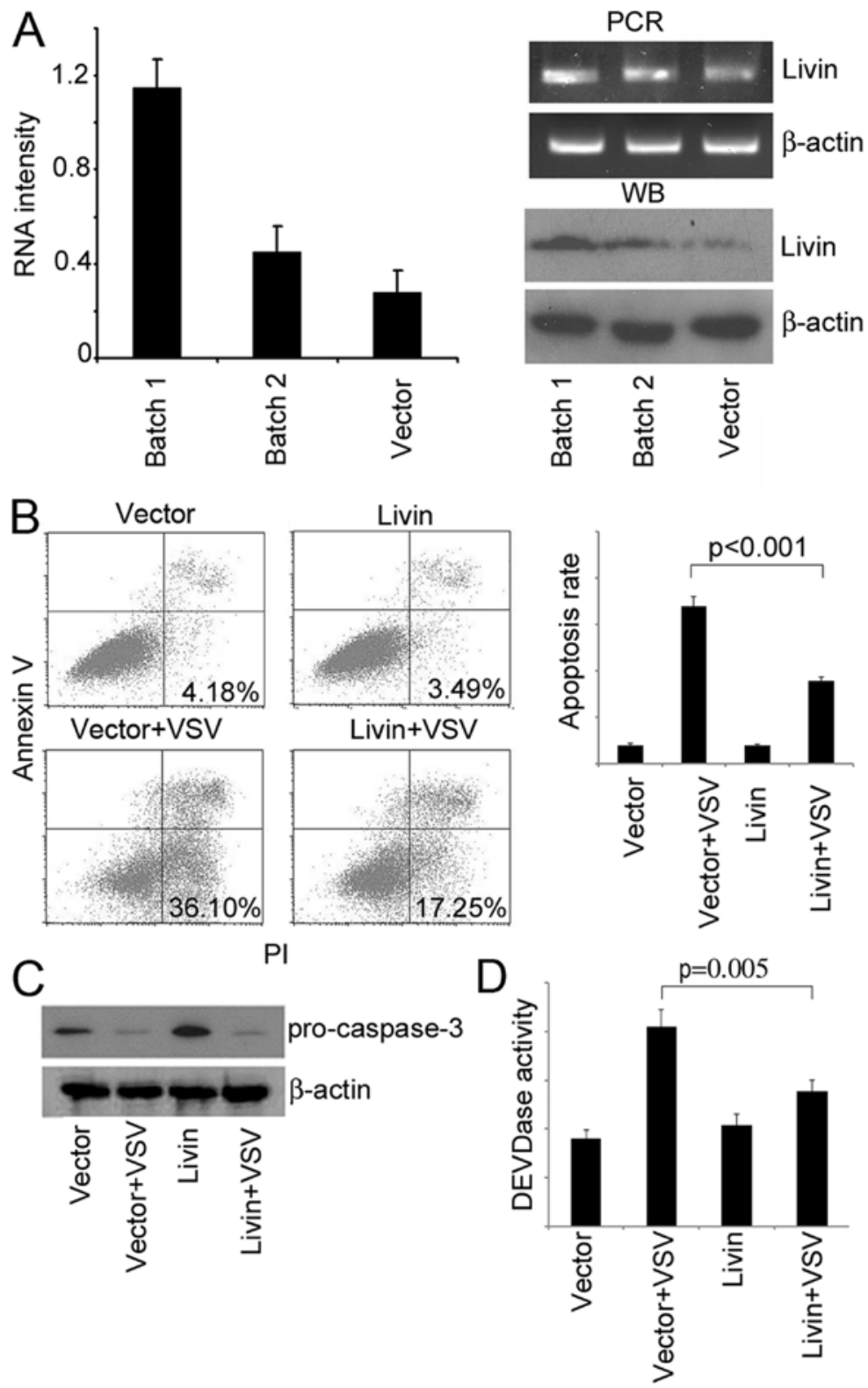

Figure 4. Two batches of stable transfectants were prepared and the expression of Livin was detected by real-time PCR, semi-quantitative PCR, and western blot analyses (A). Batch 1 with the higher expression level was selected for the following experiments. Overexpression of Livin led to reduced cell death by FCM analysis (B). Also, caspase-3 was less activated, as shown by less degraded pro-caspase-3 (C) and less DEVDase activity (D).
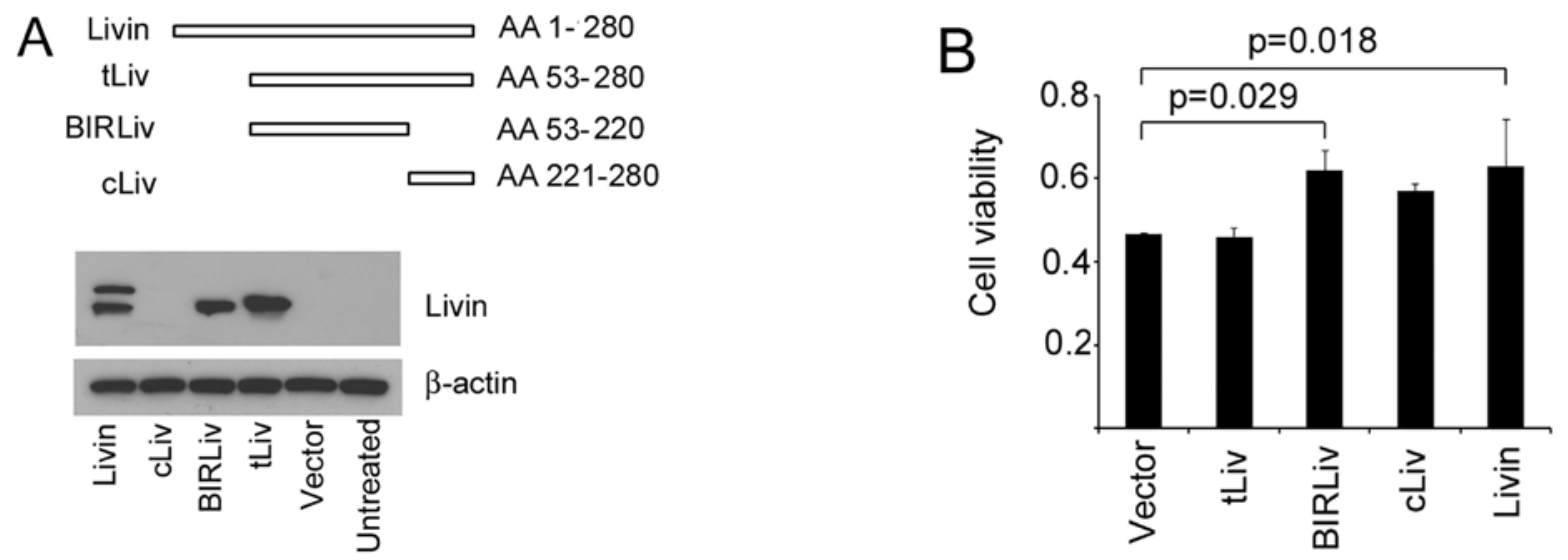

Figure 5. Schematic presentation of full-length Livin with its fragments and confirmation of their expression (A). Notably, the fragment of cLiv failed to be recognized by our antibody, possibly due to the short segment of parental Livin. However, it could be recognized by an anti-EGFP antibody at the right size (data not shown). Only the fragment of BIRLiv together with full-length Livin protected cells from VSV treatment (B). 


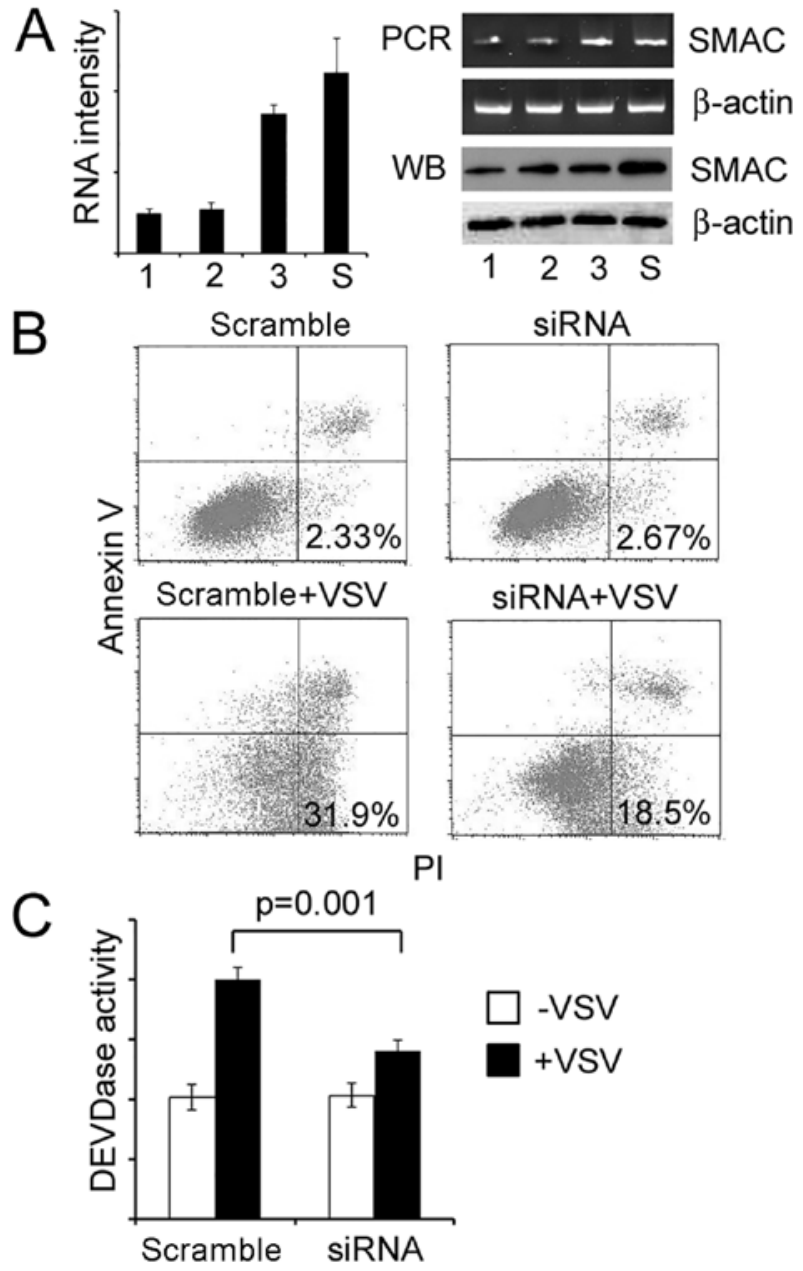

Figure 6. Three different sequences (sequence 1-3) were screened for the efficiency of RNA interference against SMAC (A). The most efficient sequence 1 was selected for the downstream experiment. Knock-down of SMAC protected the cells from VSV-induced cell death (B). Based on the observations DEVDase activity was also decreased after SMAC knockdown (C).
To further confirm that Livin was associated with SMAC, we performed a co-IP experiment. Livin bound to SMAC in both the whole-cell lysates and the cytosolic fraction (Fig. 7A). Overexpression of Livin led to a lager amount of SMAC bound in the cytosolic fraction, but not in the wholecell lysates (Fig. 7B). We next performed a co-transfection. Overexpression of Livin made cells resistant to VSV, as showed before. However, the simultaneously overexpressed SMAC compromised its anti-apoptotic effects (Fig. 7C).

Knock-down of Livin make cells vulnerable to VSV. Three pairs of candidate siRNA sequences were screened for the knockdown of endogenous Livin. The most efficient sequence 1 was selected based on results from both real-time PCR and western blot analysis (Fig. 8A) for further experiments. Knock-down of Livin made cells more sensitive to VSV treatment (Fig. 8B) with elevated caspase-3 activity (Fig. 8C). These results indicated Livin as critical for the resistance of VSV.

\section{Discussion}

VSV was proposed as a promising treatment modality for cancer (3-9). To fully realize its potential in cancer treatment, a deeper insight of its mechanisms is highly required. Previous studies focused on the IFN pathway as an explanation for its selective tumoricidal effects (11). Evasion of apoptosis is considered a hallmark of cancer cells (23), and overcoming the evasion is critical for cancer treatment (24). We believe the apoptosis pathway must play a role in mediating the therapeutic effects of VSV.

Our proposal is supported by previous reports (25-27). In one study, a small molecular BCL-2 inhibitor significantly sensitized tumor cells to VSV (28). This study highlighted that endogenous apoptosis inhibitors were associated with VSV sensitivity. The apoptosis pathway is negatively regulated by endogenous factors such as the Bcl-2 or IAP family (29).
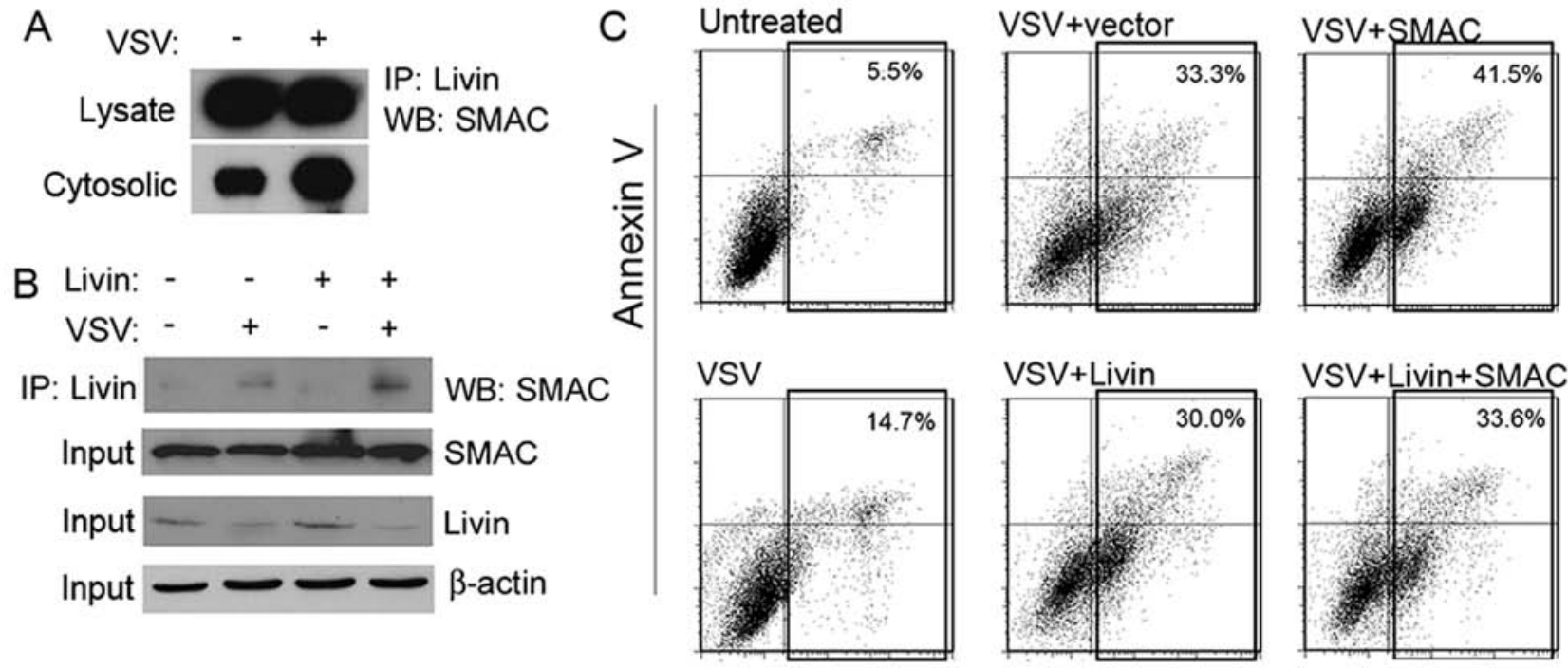

$\mathrm{PI}$

Figure 7. The binding of Livin to SMAC was confirmed by co-IP. The Livin-bound SMAC increased after treatment with VSV in the cytosolic fraction but not in the whole cell lysate (A). Overexpression of Livin led to larger amount of bound SMAC (B). Overexpression of both Livin and SMAC decresased cell death compared with SMAC alone (C). 

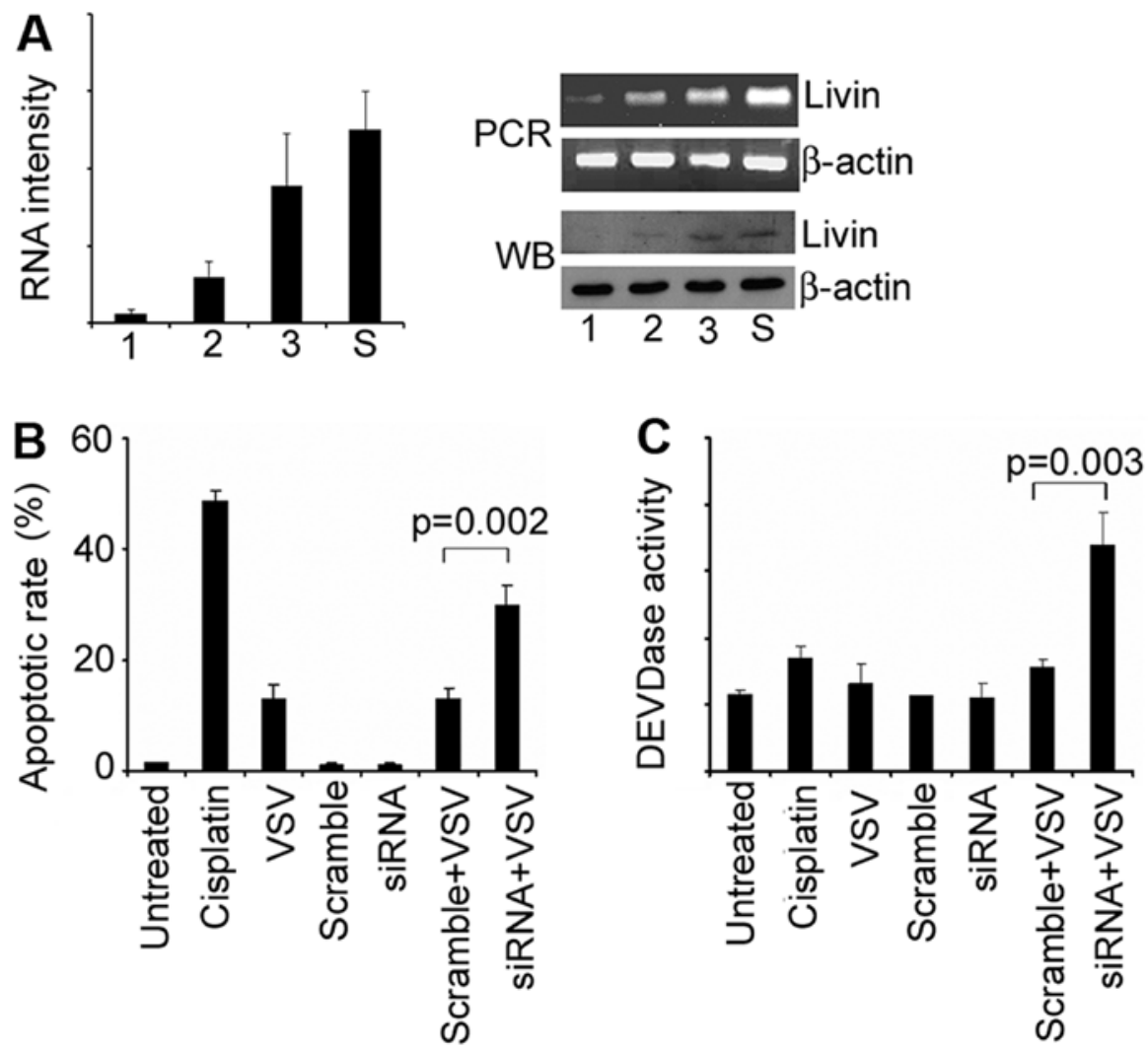

Figure 8. Three different sequences were screened for the knock-down of Livin (A). The most efficient sequence 1 was selected for the downstream experiment. Knock-down of Livin sensitized the cells to VSV treatment (B) together with elevated DEVDase activity (C).

However, few studies have been conducted to test the contribution of IAP to the apoptosis by VSV.

Previously, we reported VSV alone achieved mild effects on A549 xenogeneic transplant tumor model (6). Other groups later reported similar results. One study reported that compared with hepatocellular carcinoma Hepa-G2 cells and hepatoma Huh-7 cells, the VSV induced less apoptosis rates in A549 cells at the same MOI (30). These studies indicated lung adenocarcinoma was resistant to VSV treatment. The present study determined the expression of Livin in A549 cells to assess whether the expression of the anti-apoptotic factor Livin would contribute to the resistance to VSV in this cell line.

In our experiment, we found the Livin expression was associated with VSV infection. This suggested Livin might be involved in the VSV-induced apoptosis. As anticipated, the overexpression of Livin made cells resistant to VSV treatment. Finally, we further confirmed its role in the VSV treatment by knock-down of Livin. This emphasized Livin was important for A549 cells to resist VSV treatment. Our study shows that the members of the IAP family exert impact on the efficacy of VSV treatment.

There are controversies regarding the mechanism by which Livin inhibits apoptosis. Initially it was thought Livin could bind and suppress the downstream effector caspase-3, or -9 , which was a canonical mechanism used by other IAPs such as XIAP (13). However, later experiments failed to prove this notion. Vucic et al reported Livin only weakly inhibited caspase-9 activity with inhibition constant $\mathrm{Ki} \sim 3-5 \mu \mathrm{M}$. Also, it was argued that Livin might regulate apoptosis by sequestering SMAC from XIAP (31). In our study, we found Livin overexpression led to resistance to VSV together with reduced caspase-3 activity. We wanted to dissect the relationship between Livin and SMAC in the condition of VSV treatment. We found the knock-down of SMAC achieved similar effects with those of Livin overexpression. The co-IP also confirmed the binding of Livin to SMAC in the cytosol. The simultaneous transfection of SMAC abrogated the effects of Livin expression. These results strongly argued Livin inhibited VSV-induced apoptosis by binding and inhibiting SMAC.

We detected the binding of Livin to SMAC by the co-IP experiment. SMAC was sequestered from XIAP by the membrane of mitochondria in resting state and released into the cytosol upon apoptosis simuli (32). In our study the Livinbound SMAC increased in the cytosol but not the whole-cell lysates. We also found the expression and release of SMAC remained consistent before and after VSV treatment (data not shown). Thus, we reasoned the elevated cytosolic SMAC by the VSV was binded with Livin and lost its ability to neutralize XIAP. This might be the mechanistic explanation for the reduced apoptosis by Livin. In the whole-cell lysates, the amount of SMAC remained unchanged before and after Livin overexpression.

In conclusion, our study explored the relationship between SMAC and Livin and the impact of this relationship on the VSV sensitivity in lung adenocarcinoma cells. Our results suggested the important role of Livin and its partner molecule in the process of VSV treatment. Our study helps to deepen 
our knowledge of the molecular events after VSV transfection and improve the therapeutic effects of VSV.

\section{Acknowledgements}

This study was supported by National Natural Science Foundation of China (nos. 82172684 and 81200640).

\section{References}

1. Siegel R, Naishadham D and Jemal A: Cancer statistics, 2012. CA Cancer J Clin 62: 10-29, 2012.

2. Reck M,Heigener DF, Mok T, Soria JC and Rabe KF: Management of non-small-cell lung cancer: Recent developments. Lancet 382: 709-719, 2013.

3. Stojdl DF, Lichty B, Knowles S, Marius R, Atkins H, Sonenberg N and Bell JC: Exploiting tumor-specific defects in the interferon pathway with a previously unknown oncolytic virus. Nat Med 6: $821-825,2000$

4. Balachandran S, Porosnicu M and Barber GN: Oncolytic activity of vesicular stomatitis virus is effective against tumors exhibiting aberrant p53, Ras, or myc function and involves the induction of apoptosis. J Virol 75: 3474-3479, 2001.

5. Ebert O, Shinozaki K, Huang TG, Savontaus MJ, García-Sastre A and Woo SL: Oncolytic vesicular stomatitis virus for treatment of orthotopic hepatocellular carcinoma in immune-competent rats. Cancer Res 63: 3605-3611, 2003.

6. Li Q, Wei YQ, Wen YJ, Zhao X, Tian L, Yang L, Mao YQ, Kan B, $\mathrm{Wu}$ Y, Ding ZY, et al: Induction of apoptosis and tumor regression by vesicular stomatitis virus in the presence of gemcitabine in lung cancer. Int J Cancer 112: 143-149, 2004.

7. Lin X, Chen X, Wei Y, Zhao J, Fan L, Wen Y, Wu H and Zhao X: Efficient inhibition of intraperitoneal human ovarian cancer growth and prolonged survival by gene transfer of vesicular stomatitis virus matrix protein in nude mice. Gynecol Oncol 104: 540-546, 2007.

8. Du XB, Lang JY, Xu JR, Lu Y, Wen YJ, Zhao JM, Diao P, Yuan ZP, Yao B, Fan LY, et al: Vesicular stomatitis virus matrix protein gene enhances the antitumor effects of radiation via induction of apoptosis. Apoptosis 13: 1205-1214, 2008.

9. Stewart JH IV, Ahmed M, Northrup SA, Willingham M and Lyles DS: Vesicular stomatitis virus as a treatment for colorectal cancer. Cancer Gene Ther 18: 837-849, 2011.

10. Wong LH, Krauer KG, Hatzinisiriou I, Estcourt MJ, Hersey P, Tam ND, Edmondson S, Devenish RJ and Ralph SJ: Interferonresistant human melanoma cells are deficient in ISGF3 components, STAT1, STAT2, and p48-ISGF3gamma. J Biol Chem 272: 28779-28785, 1997.

11. Moussavi M, Fazli L, Tearle H, Guo Y, Cox M, Bell J, Ong C, Jia W and Rennie PS: Oncolysis of prostate cancers induced by vesicular stomatitis virus in PTEN knockout mice. Cancer Res 70: 1367-1376, 2010

12. Gyrd-Hansen M and Meier P: IAPs: From caspase inhibitors to modulators of NF-kappaB, inflammation and cancer. Nat Rev Cancer 10: 561-574, 2010.

13. Vucic D, Stennicke HR, Pisabarro MT, Salvesen GS and Dixit VM: ML-IAP, a novel inhibitor of apoptosis that is preferentially expressed in human melanomas. Curr Biol 10: 1359-1366, 2000.

14. Lin JH, Deng G, Huang Q and Morser J: KIAP, a novel member of the inhibitor of apoptosis protein family. Biochem Biophys Res Commun 279: 820-831, 2000.
15. Ashhab Y, Alian A, Polliack A, Panet A and Ben Yehuda D: Two splicing variants of a new inhibitor of apoptosis gene with different biological properties and tissue distribution pattern. FEBS Lett 495: 56-60, 2001

16. Tanabe H, Yagihashi A, Tsuji N, Shijubo Y, Abe S and Watanabe N: Expression of survivin mRNA and livin mRNA in non-small-cell lung cancer. Lung Cancer 46: 299-304, 2004.

17. Gazzaniga P, Gradilone A, Giuliani L, Gandini O, Silvestri I, Nofroni I, Saccani G, Frati L and Aglianò AM: Expression and prognostic significance of LIVIN, SURVIVIN and other apoptosis-related genes in the progression of superficial bladder cancer. Ann Oncol 14: 85-90, 2003.

18. Choi J, Hwang YK, Sung KW, Lee SH, Yoo KH, Jung HL, Koo HH, Kim HJ, Kang HJ, Shin HY, et al: Expression of Livin, an antiapoptotic protein, is an independent favorable prognostic factor in childhood acute lymphoblastic leukemia. Blood 109: 471-477, 2007.

19. Xiang Y, Yao H, Wang S, Hong M, He J, Cao S, Min H, Song E and Guo X: Prognostic value of Survivin and Livin in nasopharyngeal carcinoma. Laryngoscope 116: 126-130, 2006.

20. Gong J, Chen N, Zhou Q, Yang B, Wang Y and Wang X: Melanoma inhibitor of apoptosis protein is expressed differentially in melanoma and melanocytic naevus, but similarly in primary and metastatic melanomas. J Clin Pathol 58: 1081-1085, 2005.

21. Ding ZY, Liu GH, Olsson B and Sun XF: Upregulation of the antiapoptotic factor Livin contributes to cisplatin resistance in colon cancer cells. Tumour Biol 34: 683-693, 2013.

22. Liu GH, Wang C and Ding ZY: Overexpression of the truncated form of Livin reveals a complex interaction with caspase-3. Int J Oncol 42: 2037-2045, 2013.

23. Hanahan D and Weinberg RA: Hallmarks of cancer: The next generation. Cell 144: 646-674, 2011.

24. Luo J, Solimini NL and Elledge SJ: Principles of cancer therapy: Oncogene and non-oncogene addiction. Cell 136: 823-837, 2009.

25. Kopecky SA and Lyles DS: Contrasting effects of matrix protein on apoptosis in HeLa and BHK cells infected with vesicular stomatitis virus are due to inhibition of host gene expression. J Virol 77: 4658-4669, 2003.

26. Gadaleta P, Perfetti X, Mersich S and Coulombié F: Early activation of the mitochondrial apoptotic pathway in Vesicular Stomatitis virus-infected cells. Virus Res 109: 65-69, 2005.

27. Gaddy DF and Lyles DS: Oncolytic vesicular stomatitis virus induces apoptosis via signaling through PKR, Fas, and Daxx. J Virol 81: 2792-2804, 2007.

28. Tumilasci VF, Olière S, Nguyên TL, Shamy A, Bell J and Hiscott J: Targeting the apoptotic pathway with BCL-2 inhibitors sensitizes primary chronic lymphocytic leukemia cells to vesicular stomatitis virus-induced oncolysis. J Virol 82: 8487-8499, 2008.

29. Igney FH and Krammer PH: Death and anti-death: Tumour resistance to apoptosis. Nat Rev Cancer 2: 277-288, 2002.

30. Schache P, Gürlevik E, Strüver N, Woller N, Malek N, Zender L, Manns M, Wirth T, Kühnel F and Kubicka S: VSV virotherapy improves chemotherapy by triggering apoptosis due to proteasomal degradation of Mcl-1. Gene Ther 16: 849-861, 2009.

31. Vucic D, Franklin MC, Wallweber HJ, Das K, Eckelman BP, Shin H, Elliott LO, Kadkhodayan S, Deshayes K, Salvesen GS, et al: Engineering ML-IAP to produce an extraordinarily potent caspase 9 inhibitor: Implications for Smac-dependent antiapoptotic activity of ML-IAP. Biochem J 385: 11-20, 2005.

32. Wu H, Tschopp J and Lin SC: Smac mimetics and TNFalpha: A dangerous liaison? Cell 131: 655-658, 2007. 